\title{
Improving the inspection and manual cleaning of dental instruments in a dental hospital
}

\author{
Louise Campbell, Aisling Barton, Rachael Boyle, Vicki Tully \\ Dundee Dental School, Scotland
}

\begin{abstract}
Within the dental hospital setting, it is a frequent occurrence to find residual cement contaminating instruments in a newly opened kit having undergone the decontamination cycle. Any instrument found to be contaminated then cannot be used, as the area underneath the cement is not sterile. This in itself has several repercussions. These include: cross-contamination, since there is a chance that the cement will be removed and the contaminated instrument used; cost, as each new kit that will be opened due to contaminated instruments will incur decontamination costs; and finally time, which most importantly has an impact on patient experience. Our baseline data recording focussed on finding out the severity of the problem, which instruments were most affected, and how this affected patient treatment, using a questionnaire. Within the paediatric department, $27 \%$ of examination kits contained a contaminated instrument, almost one third of all kits used. This quality improvement project utilized a poster and team huddle discussions to raise awareness of the problem and successfully reduced the number of contaminated instrument kits to $7 \%$ over a period of four weeks.
\end{abstract}

\section{Problem}

Within the dental hospital setting, it is a frequent occurrence to find residual cement contaminating instruments in a newly opened kit having undergone the decontamination cycle. Any instrument found to be contaminated then cannot be used, as the area underneath the cement is not sterile. This presents a problem as the instrument must be replaced in order to be used on a patient, thus requiring a new kit to be opened. This in itself has several repercussions. These include: cross-contamination, since there is a chance that the cement will be removed and the contaminated instrument used; cost, as each new kit that will be opened due to contaminated instruments will incur decontamination costs; and finally time, which most importantly has an impact on patient experience.

\section{Background}

During our time treating patients in the dental school, we noticed that this recurring problem of contaminated instruments with residual cement was found to exist throughout all departments. We decided therefore that this would be a good opportunity to improve the quality of care, whilst also reducing unnecessary costs for the dental hospital.

Approaching our mentor, we discovered that this problem was identified around ten years ago. Since then, attempts have been made to improve this issue, but none with great success or sustainability. Within the contract between the dental hospital and central sterile services department (CSSD), visual inspection is not included, so instruments often go through the decontamination process while contaminated with residual cement. This highlights the need for an intervention within the dental hospital prior to sending the instruments to CSSD. The problem lies with the fact that manual cleaning of instruments at chairside is not occurring prior to sending the instruments for sterilisation. A paper published in the British Dental Journal in 2007 titled "Pre-sterilisation cleaning of re-usable instruments in general dental practice"[1] investigates this claim, proposing that effective cleaning prior to sterilisation is vital in the prevention of cross-infection. It states that "the cleaning of re-usable dental instruments is [also] important to ensure device longevity and functionality, removal of chemical residues and compliance with medico-legal directives." It then highlights that "effective cleaning is also vital to ensure microbial inactivation since retention of organic or inorganic debris may compromise subsequent disinfection or sterilization processes." Although this paper investigates the sterilisation of instruments in general practice setting, where decontamination is carried out at a local level as opposed to a centralised sterilisation department as utilized by the dental hospital, it demonstrates that this problem is not limited to our working environment, but has been recognised on a national level.

The Scottish Dental Clinical Effectiveness Programme[2] has released guidance on the cleaning of dental instruments, which was updated in October 2014. These guidelines come as part of the wider guidance on decontamination, and again explain that effective cleaning prior to sterilisation is required for reliable decontamination of instruments. It states that "any organic material or adherent dental materials left on instruments can inhibit these processes [disinfection and sterilisation]. This can also cause corrosion of instruments or impair their function, and might lead to transmission of infection from one patient to another."

We were able to study existing data collected within the children's department of the dental hospital[3] by Dr Daffyd Evans, showing that $36.7 \%$ of all instrument kits opened were not free from cement contamination. The study also showed the instruments most likely to be contaminated with cement. These included: flat plastic, excavator, burnishers, and thymosin probe. However these were 
not the only instruments shown to have contamination, therefore there is a risk to all instruments of being contaminated.

From our search we are unaware of any other improvement projects tackling this issue.

See supplementary file: ds4034.pdf - "Audit of Hand Instrument Kits RESULT"

\section{Baseline measurement}

To collect our baseline data, we used the three key phases of baseline measurement: develop, collect, analyse. Our main objective for this project was to investigate whether contamination of instruments is a significant problem, and to what extent. Our first process measure that we assessed was the number of kits that were contaminated on opening. The second process measure was to look at which instruments were affected. The outcome measure was whether the contaminated instruments affected patient care.

In order to collect the data, we had to decide on a universal definition of contamination. We defined a contaminated instrument as one with any amount of visible cement and explained this to our colleagues who assisted in collecting the data, during a clinical session in the children's department of the dental hospital. We decided to collect our data using a short questionnaire (attached below) which was our first PDSA cycle.

PDSA 1 - A Questionnaire to Investigate the Problem

P- Prepare a questionnaire, which would highlight whether contaminated instruments were a problem, and which instruments were commonly affected. The questionnaire should be simple, and not time-consuming, to encourage students and staff to participate in our improvement project.

D- During a clinical session in the children's department of the dental school, every student treating a patient was asked to examine their kit on opening and identify any contaminated instruments. We asked the students to specify which instrument(s) was/were contaminated and if this affected patient care.

S- The students found the questionnaire easy to understand and every student who treated a patient completed a questionnaire. The feedback also highlighted that they were simple and quick to complete which encouraged participation. Of the six kits that were opened on the afternoon clinic, four were free from cement contamination, while two kits were not free from cement. Of these two kits, one had a contaminated flat plastic and treatment was not affected. The other contaminated kit contained a contaminated excavator, which did affect treatment and compromise patient care.

We realized from this first cycle that we did not have all of the information we required. We knew that the contamination affected patient care, but we did not know why.

A- We decided to update the questionnaire to include how the care of the patient was affected, and carry out a second PDSA cycle.
PDSA 2 - A Questionnaire to Assess How our Problem Impacts upon Patient Care

On carrying out our second PDSA cycle with the updated questionnaire, we received nine returned forms, of which two students had reported contaminated instruments, whilst the other seven found no residual cement in their kits. Although we anticipated more detailed results in this second cycle with regards to how the contaminated instruments affected patient care, our responses stated that there was in fact no effect on patient care in this clinical session. The feedback we received from our colleagues regarding this was that the instruments that were contaminated were not required for that particular treatment, therefore had no impact. However, if another procedure was to be carried out that required these instruments, then an impact on patient care would have been reported. In the first case the contaminated instrument was reported as the flat plastic, and in the second case, the excavator was contaminated. This highlights that these instruments are the most commonly affected and that an intervention is required.

The results that we collected during our baseline data collection, demonstrated that our results correlated with the previous data collected within the Paediatric department at $27 \%$ of examination kits containing a contaminated instrument, almost one third of all kits used.

See supplementary file: ds3675.docx - "QI Project Tool Version 1"

\section{Design}

Our first step in tackling the problem was to assign responsibility to the person using the instrument kit, so that our intervention was targeted to this individual. We had several discussions with our senior clinician, head dental nurse, and patient safety lead to ensure that our intervention would be viable. One suggestion which arose at these meetings was the idea of introducing single use instruments, which would eliminate the problem. However, we were intent on avoiding this approach as we felt that although it was financially viable, the constant disposal of instruments would be detrimental to the environment. Although the single use instruments may seem to be cost effective on the surface, we also anticipated there would be hidden costs involved, such as having to increase the quantity of sharps bins for their safe disposal.

Therefore we came to our second approach, which would be to safely manually clean the instruments that we already have. This raised concerns in terms of sharps injury risk, and risk of damaging the surface of metal instruments. It became clear that we would have to devise a safe procedure for doing so, which involved working closely with the infection control team to gain approval. Taking advice from our senior clinician and our patient safety lead, we concluded that the safest and most cost-effective process would be to utilise detergent wipes and wooden spatulas as required, in order to remove contaminants from instruments prior to sterilisation. This is a process that had not been trialled in NHS Tayside so far; the NHS Tayside Infection Prevention and Control Policy states that the decontamination for dental instruments should take place at 
CSSD, or otherwise in an appropriate LDU, no mention of manual cleaning is provided.[4] Once this process had been approved, we deduced that the most effective way to explain this process to our colleagues would be to present it as a poster. Included in our poster, we realised it should have a clear flow diagram as to how the process should be carried out, and also briefly explain the logic behind our intervention. Please see attached poster.

We created a process map tracking an instrument kit's journey following use, to not only explain our intervention to other stakeholders, but also to illustrate where our intervention would fit in on this journey, and have the most effect. Please see attached process map.

On reflecting on previous unsuccessful attempts within the department to tackle this problem, we were aware from our quality improvement knowledge that education alone may not obtain the desired result. Therefore to increase motivation to comply, we devised a method of tracing individual kits back to the user, therefore improving accountability of practice. This method involved recording the date, time, student number/staff name, and the bar code number of the kit used. We decided that this would not be a long term component of our intervention, but a tool to aid us in implementing it and help to establish the process as part of every day practice.

\section{Strategy}

Our first step aimed to test the effectiveness of our designed poster, and assess whether it was clear enough to explain the process of manual cleaning effectively. This would be essential to allow us to meet our main aim of every dental student/staff member being compliant with the manual inspection and cleaning of instruments within the paediatric department of the dental school within four weeks.

\section{PDSA 3 - Designing our Intervention Poster}

Our third PDSA cycle involved testing the poster with one student and asking them for feedback as to how easy the process was to follow. We expected that the student would find the poster informative and self explanatory. However, the findings of this test showed that some additional verbal information was required. This allowed us to modify how we planned to deliver the intervention, ensuring that the poster was explained verbally at each team huddle at the beginning of clinic, as well as being displayed throughout the clinic to be viewed easily by all students and staff working in the area. This would be the basis of our next test, to assess whether this approach would achieve full understanding. Another useful outcome of this PDSA cycle, was feedback from the student recommended specific locations in the clinic that they felt would be most noticeable and effective. Through the discussions of this test, we decided that the posters would be placed on the wall over the 'dirty instrument' trolley to serve as a reminder, centrally within the clinical area, and within the tutorial room so that it would be easily accessed by students to read up on the process.

PDSA 4 - Testing our Intervention
The fourth PDSA cycle was carried out on a morning clinic within the department. At the team huddle at the beginning of clinic (at which all members of the team were present, including students, staff, and nurses) the intervention was described by displaying the poster and supplementing this with verbal information to further explain the problem and our intervention. Feedback from the group highlighted that this was a more effective method, and full understanding of what was expected was demonstrated. We decided that our intervention was now ready to be spread within the department, and PDSA 5 would involve carrying out this process at morning and afternoon clinics for two weeks.

PDSA 5 - Implementing our Intervention

PDSA 5 involved utilizing the information we had collected in the previous tests, displaying the poster around the clinic in key areas, explaining the process at the team huddles, and ensuring that all students/staff using examination kits were carrying out inspection and manual cleaning as necessary, before returning the kit to the "dirty" trolley. Accountability of practice was introduced by recording the clinical session, barcode of kit used, and student number/staff name. This meant that when the instrument kits were examined for a second time by a dental nurse before sending to CSSD, if a contaminated instrument was present, this could be traced back to the individual. Please see attached flow diagram which depicts the journey of an examination kit through this process.

See supplementary file: ds5597.docx - "Intervention Poster"

\section{Post-measurement}

As our intervention was tested, implemented, and spread, we collected data to prove its reliability. After the planned two weeks of implementing our intervention, our results showed that every examination kit had been through the process at least once, and all were free from contaminants. We were able to ensure this using the method of placing a sticker on the outer packaging following delivery of the sterilised kits from CSSD. The method of having the examination kits double-checked before sterilisation and the barcode traceability is what proved that we did not have any contaminated kits, as no bar codes required following up during the two week process. This highlighted that awareness of the problem had increased and staff and students were complying with the inspection and manual cleaning.

In the following two weeks, we used an almost identical data collection method to our baseline data collection. Throughout these two weeks, the recording of bar codes was stopped. This was due to the fact that we felt too much paperwork was causing resentment towards the project, and that at this stage it would be more beneficial to collect physical data as we had during our baseline data collection. This was in the form of a simple questionnaire that asked whether or not any instruments in the newly-opened kit were contaminated, and if so, which instruments and whether this had an impact on patient care. (See attached.)

Our data collection targeted the same group as our baseline collection, which was any member of staff or student who had used 
an examination kit. Over the period of two weeks, we received 28 completed questionnaires. This number was lower than we expected, however this could be due to the fact that we were not present for one of the weeks so were not encouraging or reminding people to fill them out. It may also be down to failed appointments which is a regular occurrence in the paediatric department, therefore reducing the number of patients seen and so the number of kits used. This is especially true as one week coincided with school holidays so less appointments were booked/attended on this week.

However, of the 28 questionnaires we received, only two reported a contaminated instrument. Both of these claimed to have an impact on patient treatment. We found that $7.1 \%$ of our kits were contaminated, this being an improvement from our baseline measurement which was $27 \%$ of examination kits having a contaminated instrument. Although we have achieved a reduction, it has to be questioned why the number is still at $7 \%$, as in our first two weeks of the intervention, we had reduced it to zero. This puts into question the sustainability of the intervention and what needs to be improved so that we can continue to solve the problem without our presence on clinic being necessary.

See supplementary file: ds6576.pdf - "Exam kit journey \& run chart"

\section{Lessons and limitations}

There were a few limitations that we encountered when carrying out this project, all of which stem from the fact that dentistry is a demanding course in itself which does not leave much time for carrying out an improvement project on top of studies and clinical practice. Due to our need for gaining experience in all clinical areas, we have only a set amount of time in each specific department. Therefore we could not always be present in the Paediatric department to take part in the running of the intervention due to other clinical commitments. This made organising the intervention more difficult, and also made sustainability more of a challenge. This has made us realise how important team-working is in quality improvement, especially delegating tasks to other members of the team. For example, this project could not have been possible without the input and cooperation of the dental nurses, who allowed the intervention to run in our absence. It is therefore our hope that the intervention will be continued as we move into our final year when our time in the dental school is minimal.

Another limitation was the lack of awareness of quality improvement within the dental school. This is something that will only increase with time and development of more quality improvement projects by the students. We would hope that with increasing awareness, students will realise the impact they can have on improving patient care and engage more fully in the projects of fellow students.

Furthermore, similar to the above limitation of lack of awareness, we also lacked the presence of a clear tutor within the dental school with knowledge of quality improvement to guide us throughout the improvement project. Although we had a great deal of help from the senior clinician in the paediatric department, his retiral occurred before the intervention had begun. However, during the implementation of our intervention we received a great deal of support from the head dental nurse, whose input was invaluable.

\section{Conclusion}

As our first experience of quality improvement, we are pleased as to how the project has run, and have learned a great deal from the experience. We are proud of the success we have achieved, not only in terms of reducing the number of contaminated instruments but also raising awareness of the problem. We feel that at this point, the intervention is not sustainable, but as the process becomes habit and new generations of students come through the system, we feel that it has the potential to be. At present, the department are trialling the use of disposable instruments as an alternative to our intervention. This removes the problem of dependency on students and staff carrying out the inspection and manual cleaning process.

We also feel that by piloting the first quality improvement project within the dental school, we have taken the first step in raising awareness of quality improvement itself, both with staff and students.

\section{References}

1 Bagg J, Smith AJ, Hurrell D, McHugh S, Irvine G. Pre-sterilisation cleaning of re-usable instruments in general dental practice. British Dental Journal 2007; 202():

http://www.nature.com/bdj/journal/v202/n9/full/bdj.2007.124.html (accessed 24 June 2015).

2 Scottish Dental Clinical Effectiveness Programme. Decontamination. Dundee Dental Education Centre, Frankland Building, Small's Wynd, Dundee DD1 4HN: ; 2014. www.sdcep.co.uk (accessed 24 June 2015).

3 Data collected by Daffyd Evans, Honorary Senior Lecturer in Paediatric Dentistry, Dundee Dental Hospital \& School.

4 Infection Control Team. NHS Tayside Infection Prevention and Control Policy.

http://www.bbvmcntayside.scot.nhs.uk/Professionals/Guidelines/BB V\%20Guidelines/Infection\%20Control\%20June\%2012.pdf (accessed 24/11/15)

\section{Declaration of interests}

Nothing to declare.

\section{Acknowledgements}

Vicki Tully, Wendy Roud, Daffyd Evans, Marilla Hunter

\section{Ethical approval}

Ethics not required as this project was undertaken as a service 


\section{BMJ Quality Improvement Reports}

improvement. 